Annuaire suisse de politique de développement

$1 \mid 1981$

Annuaire Suisse - Tiers Monde 1981

\title{
La nouvelle contestation de la coopération suisse au développement
}

Die Neue Bestreitung der Schweizerischen Entwicklungszusammenarbeit

Jacques Forster

\section{(2) OpenEdition}

1 Journals

Édition électronique

URL : http://journals.openedition.org/aspd/1092

DOI : 10.4000/aspd.1092

ISSN : 1663-9669

Éditeur

Institut de hautes études internationales et du développement

Édition imprimée

Date de publication : 1 janvier 1981

Pagination : 213-222

ISSN : 1660-5934

\section{Référence électronique}

Jacques Forster, "La nouvelle contestation de la coopération suisse au développement », Annuaire suisse de politique de développement [En ligne], 1 | 1981, mis en ligne le 18 janvier 2013, consulté le 08 septembre 2020. URL : http://journals.openedition.org/aspd/1092 ; DOI : https://doi.org/10.4000/ aspd. 1092 


\title{
LA NOUVELLE CONTESTATION \\ DE LA COOPERATION SUISSE AU DEVELOPPEMENT
}

\author{
Jacques Forster
}

\begin{abstract}
DIE NEUE BESTREITUNG DER SCHWEIZERISCHEN ENTWICKLUNGSZUSAMMENARBEIT
\end{abstract}

Zusammenfassung

Seit dem Herbst 1980 sieht sich die DEH (Direktion für Entwicklungszusammenarbeit und humanitäre Hilfe) einer neuen Art von Kritiken ausgesetzt. Sie kommt aus politischen und beruflichen Kreisen, welche sich bisher zu diesem Teil der Regierungspolitik kaum öffentlich äusserten. Seit mehreren Monaten konzentrieren sich diese Kritiken auf die geplante N.O. (Neue Organisation).

Eine genaue Analyse zeigt jedoch, dass siz darüber hinaus wesentliche Aspekte der schweizerischen Entwicklungspolitik angreifen. Die hauptsächlichsten Kritiken betreffen die Verwaltung der Entwicklungszusammenarbeit, ihre Transparenz und ihre Zusammenarbeit mit der Privatwirtschaft.

Die Forderung, die Privatwirtschaft mehr an der Durchführung der Entwicklungszusammenarbeit zu beteiligen, erhält von zwei Seiten Auftrieb. International gesehen erleben wir einen Aufschwung der liberalen Wirtschaftsdoktrin. In der Schweiz selber stehen wachsende Kredite für die Entwicklungszusammenarbeit zur Verfügung. Es stellt sich erneut die Frage, welchen Platz die schweizerische Entwicklungszusammenarbeit in unserer Aussenpolitik einnehmen soll.

1. Depuis l'automne 1980, un phénomène nouveau est apparu sur la scène politique suisse concernant la coopération de notre pays avec les pays du Tiers Monde : des critiques répétées sont adressées au service compétent de l'Administration fédérale, la DDA (1), portant aussi bien sur la qualité de son travail et la compétence de ses collaborateurs, que sur la politique qu'elle poursuit en application de la loi fédérale sur la coopération au déve- 
loppement et I'aide humanitaire internationales entrée en vigueur en 1977. Ce n'est certes pas la première fois que la DDA fait l'objet de critiques; ce qui est nouveau, c'est qu'elles émanent de milieux politiques et professionnels - élus des partis radical et démocrate-chrétien, représentants de firmes d'ingénieurs-conseils - qui jusqu'ici ne s'étaient guère manifestés, publiquement, sur ce chapitre de l'action gouvernementale.

Cette nouvelle contestation de la politique suisse de coopération au développement s'est largement exprimée par le biais d'interventions parlementaires, de débats télévisés ainsi que dans la presse. II faut remonter à 1976, à la campagne précédant le vote populaire sur l'octroi d'un crédit de 200 millions de francs à une institution du groupe de la Banque mondiale, I'IDA, pour trouver dans les les médias un intérêt aussi intense pour la coopération au développement poursuivie par les autorités helvétiques.

Dès les premiers mois de 1981, les critiques se sont concentrées sur une proposition de la DDA de mettre sur pied avec les plus importantes œuvres suisses d'entraide un nouvel organisme de coopération au développement. Il s'agissait de créer, sous la forme d'une association, une "nouvelle organisation" (provisoirement baptisée N.O.) chargée de préparer et de réaliser les projets de développement "à la demande et sur instructions de la Confédération et financés par celle-ci" (2), en particulier dans le domaine complexe du développement rural.

La gestion de tels projets requiert un personnel dont la DDA ne dispose pas vu le blocage des effectifs des fonctionnaires fédéraux. Cette N.O. emploierait une dizaine de collaborateurs et gérerait de 12 à 20 millions de francs par année, ce qui représenterait au maximum $3 \%$ de l'aide publique suisse au développement.

A première vue, la campagne lancée contre cette initiative de l'Administration fédérale ne représente qu'une tempête dans un verre d'eau, concernant un point relativement secondaire de la politique gouvernementale de coopération au développement. Mais une analyse du contenu des critiques et du contexte dans lequel cette campagne a été lancée laisse supposer que l'enjeu du débat se situe bien au-delà de la création de la N.O. pour toucher des aspects fondamentaux de la politique suisse de coopération au développement. 
Ce débat marque sans doute le début d'une nouvelle attitude des milieux économiques à l'égard d'un élément important de notre politique intérieure. Il illustre aussi la première rupture sérieuse du consensus adopté par les partis gouvemementaux à l'égard de la coopération et qui s'était manifesté notamment par l'adoption de la loi de 1977, le soutien au crédit de 200 millions de francs à I'IDA contre lequel un référendum avait été lancé, et plus récemment en 1980, l'acceptation d'un crédit cadre de 1.650 millions de francs pour la poursuite de la coopération technique et financière.

C'est cette nouvelle situation que nous tentons d'analyser ci-après.

2. En automne 1980, les commissions des affaires étrangères des Chambres fédérales sont informées du projet de création de la N.O. à l'occasion de la discussion du nouveau crédit de programme destiné à la coopération technique et à l'aide financière; en septembre, la Commission consultative pour la coopération au développement discute ce projet et approuve la poursuite de son étude. Une description détaillée du projet paraî́t dans le quotidien bernois "Der Bund" le 19 septembre 1980 (3).

Mais certains milieux proches des firmes d'ingénieurs-conseils s'inquiètent : cette nouvelle institution ne ferait-elle pas concurrence aux entreprises du secteur privé; sa création n'est-elle pas symptomatique de la réticence qu'éprouverait la DDA à collaborer davantage avec les firmes d'ingénieurs-conseils? Telles sont les interrogations qui transparaissent dans les interventions de parlementaires et de représentants de ces firmes (4).

Dans sa réponse, à une question du Conseiller national Lüchinger, le Conseil fédéral (5) assure que la N.O. n'empêchera pas le maintien, voire le renforcement, de la collaboration entre la DDA et les firmes d'ingénieurs-conseils. Il souligne en outre que les projets d'aide financière - en particulier la réalisation d'infrastructures économiques et sociales - se prêtent mieux à une collaboration avec les firmes d'ingénieurs que les projets de coopération technique qui requièrent un type de collaboration avec la population que ces firmes ne pratiquent pas. "Nous sommes convaincus" dit finalement le Conseil fédéral "qu'à l'avenir, les entreprises 
suisses auront, dans le cadre de l'aide financière, plus de possibilités de travailler pour des projets de développement".

Mais les assurances données par le Conseil fédéral ne satisfont pas les intéressés. Au contraire! L'affirmation du gouvernement selon laquelle les firmes sont peu aptes à collaborer étroitement avec les groupes de population bénéficiaires de la coopération technique et à encourager leur auto-développement avive la controverse sur les mérites respectifs des projets des entreprises privées et ceux de la coopération au développement.

Dès lors, la critique s'élargit, le débat devient public (6). Ce n'est plus seulement la N.O. qui est en cause mais le style de travail de la DDA, la compétence de ses collaborateurs, son attitude jugée hostile à l'économie privée (7). Devant cette levée de boucliers, la DDA décide de repousser de quelques mois la création de la N.O. Elle répond ainsi au vœu exprimé par la nouvelle Commission consultative pour la coopération au développement - nommée au début de 1981 - qui avait à nouveau ouvert ce dossier à l'initiative du Conseiller national radical zurichois De Capitani. Il est en outre annoncé, que pour des raisons juridiques, la Confédération ne sera pas membre de la N.O. Peut-être espère-t-on à Berne désamorcer ainsi la controverse.

Parallèlement à la polémique sur la N.O. d'autres interventions témoignent du mécontentement de certains partis politiques à l'égard de la gestion de la coopération au développement. Mentionnons-en deux :

- En décembre 1980, le Conseiller aux Etats d'Appenzell Rhodesintérieures, le démocrate-chrétien Carlo Schmid, dépose une motion visant à accroítre la participation du Parlement aux décisions concemant l'aide au développement en proposant que ce dernier se prononce annuellement sur "les mesures que la Confédération envisage de prendre sur le plan de la coopération technique, de l'aide financière et de l'aide humanitaire ordinaire". La motion demande en outre que la loi sur la coopération au développement soit modifiée dans ce sens. Cette motion est largement interprétée comme un geste de méfiance à l'égard de la DDA, sans cependant que les raisons de cette méfiance apparaissent encore clairement (8). 
- Au printemps est créé un groupe parlementaire Suisse - Tiers Monde, ne comprenant que des représentants de partis bourgeois, dont le but est, selon ses membres, de favoriser une utilisation aussi efficace que possible de l'aide suisse au développement, d'améliorer l'information dans ce domaine, de promouvoir une collaboration plus étroite entre l'économie, les responsables de I'aide au développement et le Parlement. Le groupe lors de sa séance de fondation s'inquiète de la façon dont seront utilisés les nouveaux crédits de coopération au développement et du projet de la DDA de créer une nouvelle organisation (9).

Il est probable que "I'affaire" de la N.O. ait permis la cristallisation de critiques et de revendications des milieux économiques à l'égard de la politique officielle de coopération au développement. Essayons de cerner et d'expliquer les unes et les autres.

3. Il faut tout d'abord évoquer la crainte exprimée par des représentants des firmes d'ingénieurs-conseils (cf. note 4) que la N.O. fasse concurrence au secteur privé. La faible dimension prévue pour la N.O. par rapport à une branche comptant environ 20.000 collaborateurs fait que cette crainte est infondée, ceci d'autant plus que la N.O. travaillerait dans des domaines - par exemple : développement rural à la base, soutien d'initiatives de communautés locales - dans lesquels les firmes en question ne sont pas spécialisées.

Au-delà de la N.O., les critiques adressées à la DDA ont trait à l'efficacité de sa gestion, à la transparence de ses activités et à son attitude à l'égard d'une collaboration avec les entreprises du secteur privé en Suisse. Reprenons ces différents points.

a) Au chapitre de la gestion, il a été dit que la préparation et la réalisation des projets ne sont pas faites d'une façon satisfaisante, d'une part parce que les collaborateurs de la DDA ne sont pas assez expérimentés, et d'autre part parce qu'une administration publique est moins efficace qu'une entreprise privée (10) .

II serait sans doute intéressant de pouvoir comparer l'efficacité des projets d'aide publique au développement et de ceux de l'économie privée. Encore faudrait-il énoncer clairement au 
préalable quels sont les critères d'évaluation et arrêter un échantillon représentatif de projets à examiner.

En comparant des investissements directs privés et des projets de la coopération publique au développement, il conviendrait aussi de tenir compte des conditions très différentes dans lesquelles se réalisent les uns et les autres. Les investissements directs ne se réalisent que si les études préalables font apparaître que les conditions de réussite financière sont réunies. Ils se dirigeront donc de préférence vers des régions équipées en infrastructures (transport, énergie, etc.), disposant de maind'œuvre qualifiée, jouissant de conditions naturelles favorables (climat, sols, etc.). C'est dire qu'ils s'implanteront dans des zones qui, à des titres divers, sont relativement privilégiées.

La coopération publique au développement doit, selon la loi, soutenir en priorité les régions et groupes de population les plus défavorisés. Sa vocation est donc de réaliser ses projets dans des régions où les conditions sont, par définition, plus difficiles. D'autre part, l'objectif du projet de coopération n'est pas de rentabiliser à court ou moyen terme un investissement, mais - toujours selon la loi - d'accroître la capacité d'un pays ou d'une région d'assurer son propre développement. II est donc bien téméraire de vouloir comparer les prestations publiques et privées dans ce domaine.

Pour ce qui est des travaux accomplis dans le cadre de la coopération technique, on constate que la DDA ne réalise ellemême que des projets complexes, avant tout dans le domaine du développement rural, et qu'elle délègue largement la réalisation de tâches spécialisées dans ces projets ou des projets plus ponctuels à des spécialistes mis à disposition par des œuvres d'entraide privées, des instituts de recherche et... des entreprises privées. La diversité des tâches accomplies par les secteurs privé et public, qui ont chacun leur spécialisation, rend les comparaisons particulièrement hasardeuses, voire dénuées de sens.

b) Un autre problème a été fréquemment évoqué, celui de la transparence de la gestion de la DDA et du contrôle parlementaire de ses activités. Actuellement, la situation est la suivante : le Parlement adopte les crédits-cadres de coopération au déve- 
loppement sur la base d'un Message fort détaillé qui décrit et chiffre les différentes composantes des programmes de coopération (coopération technique, aide financière, aide multilatérale, bilatérale). De plus, le Parlement adopte chaque année le budget de la Confédération dans lequel figurent les crédits annuels destinés à la coopération au développement. Enfin, de par sa fonction de contrôle, le Parlement peut exiger des renseignements précis sur tout aspect des activités de la DDA. La coopération au développement est donc soumise aux mêmes contraintes et contrôles que les autres activités de la Confédération. On ne voit guère quels sont les arguments qui permettraient d'en faire un cas particulier.

c) Dernière critique : la DDA serait réticente à l'égard d'une collaboration avec l'économie privée. Cette attitude serait due à des collaborateurs de la DDA accusés d'être des "idéologues du développement", sans expérience du terrain ou de l'entreprise privée.

Cette critique est curieuse. Des chiffres publiés en mai 1981 montrent que pour 406 millions de francs d'aide publique au développement, 540 sont revenus dans l'économie privée suisse. C'est dire que pour 1 franc versé par le gouvernement, les entreprises suisses reçoivent 1,33 franc de commandes. "La Banque mondiale, à elle seule, passe des commandes à la Suisse pour 200 millions de francs. Les grandes banques régionales de développement, le Programme des Nations Unies pour le développement donnent également plus de commandes à la Suisse qu'ils ne reçoivent d'argent" (11). Sur le plan bilatéral, les entreprises suisses reçoivent commandes et mandats par le biais de crédits mixtes, d'études ou de réalisation de projets dans certains secteurs comme par exemple celui de la formation hôtelière. Globalement donc, l'économie suisse profite largement des activités liées à la coopération au développement et de la politique gouvernementale dans ce domaine. La critique ne résiste pas à l'examen des chiffres.

Il ne faut d'ailleurs pas oublier que sur ce point précis d'autres critiques de la politique gouvernementale lui reprochent une "aide" trop égoïste, se traduisant précisément par l'importance des flux en retour. 
4. Les entreprises suisses désirent accroître leur participation à la réalisation de projets de coopération au développement. Cette revendication - sans doute ancienne - s'exprime aujourd'hui avec force sous l'effet de deux facteurs nouveaux de caractères différents :

Sur le plan idéologique d'abord, nous vivons une phase nouvelle marquée par une renaissance des doctrines inspirées par un extrême libéralisme économique. L'arrivée au pouvoir de Margaret Thatcher en Grande-Bretagne et surtout de Ronald Reagan aux Etats-Unis ont, dans beaucoup de pays, renforcé la confiance des secteurs socio-économiques qui ressentent comme une menace le rôle croissant de l'Etat dans la vie économique des entités nationales. En Suisse, ce courant s'est aussi manifesté, bien que le rôle de l'Etat y soit moins important que dans d'autres pays européens. "Plus de liberté, moins d'Etat", ce slogan d'un grand parti politique lors des élections fédérales de 1979 en témoigne, ainsi que la motion d'un radical argovien, le Conseiller national Hunziker, qui souhaite confier à l'économie privée des tâches actuellement réalisées par les pouvoir publics. Certains des critiques de la DDA ne veulent pas autre chose pour les tâches de coopération au développement.

Les milieux de l'économie prennent maintenant l'initiative dans un domaine où, dans les années 1970, ils se sont bornés à réagir aux attaques d'organisations tiers-mondistes suisses qui critiquaient la politique des multinationales commerciales, industrielles et bancaires dans les pays du Tiers Monde. Aujourd'hui, les porte-parole de ces milieux sortent de leur réserve habituelle pour mettre en cause une politique gouvernementale qui à leurs yeux est trop éloignée de la traditionnelle politique économique extérieure de la Suisse axée sur la recherche de débouchés pour ses marchandises et d'occasions de placement pour ses capitaux.

Sur le plan économique, il est probable que l'accroissement des crédits de coopération au développement survenu ces dernières années explique, en partie du moins, le nouvel intérêt des milieux économiques pour la coopération au développement. Le chiffre de 1.650 millions de francs - montant du dernier crédit-cadre de coopération au développement - a pu donner à certains entrepreneurs la conviction que la coopération au développement pouvait devenir une source intéressante de mandats et de commandes. 
Dans cette optique, la proposition de créer la N.O. a immédiatement été perçue comme exprimant une tendance dangereuse, dans une conjoncture où l'obtention de contrats à l'étranger devenant plus ardue qu'au début des années 1970 (12).

Au moment où ce texte est rédigé, nous ignorons encore si la N.O. sera créée ou non. Quoi qu'il en soit, la controverse à son propos aura, une fois de plus, soulevé le problème de la place de la coopération au développement dans notre politique extérieure. S'agitil, comme le veut la loi y relative, de "soutenir les efforts des pays en développement aux fins d'améliorer les conditions de vie de leurs populations... et tendre ainsi à long terme vers un meilleur équilibre au sein de la communauté internationale" (13) ? Ou s'agit-il d'en faire un instrument de la politique économique extérieure visant à renforcer la position des entreprises helvétiques comme l'indiquent certaines prises de position évoquées dans cet article?

Bien qu'elle ait été engagée sur des questions touchant aux modalités de la coopération suisse au développement, la controverse que nous avons évoquée peut donc avoir des conséquences sur les objectifs et la pratique de cette coopération. II est donc nécessaire que, face à l'opinion publique, les options soient clairement exposées et que le débat porte ouvertement sur la seule question qui à moyen et à long terme importe vraiment : comment aménager nos relations économiques avec les pays du Tiers Monde dans un sens qui favorise le développement de ces pays et conforme aux objectifs de la loi fédérale sur la coopération au développement (14).

\section{Notes}

1. Direction de la Coopération au développement et de l'aide humanitaire internationales du Département fédéral des affaires étrangères.

2. Un portrait de la N.O. a été publié dans le numéro 9 de juin 1981 de la Revue Entwicklung-Développement (pp. 38 à 41) éditée par la DDA et l'OFAEE. 
3. Voir également ci-dessus le chapitre de la "Revue" sur la coopération technique et l'aide financière.

4. Voir notamment :

- L'interpellation du Conseiller national radical de Zurich, Lüchinger, qui, à propos de la N.O., demande si le Conseil fédéral "est disposé à associer aussi largement que possible les firmes suisses d'ingénieurs-conseils existantes à la réalisation de l'aide au développement" (question ordinaire Luchinger 80.785 du 10 octobre 1980).

- L'article de H.C. Von Schulthess, directeur de I'USSI

(Association suisse des firmes d'ingénieurs-conseils et d'architectes), dans la NZZ du 13 mars 1981.

5. Réponse du 26 novembre 1980 - Bulletin officiel de l'Assemblée fédérale - Conseil national 1980, pp. 1713-1714.

6. De nombreux articles attaquant la N.O. et la DDA paraissent dans la presse, en particulier la NZZ, à partir du mois de mars.

7. Traduction libre de l'expression allemande beaucoup entendue dans ce contexte de "Wirtschaftsfeindlichkeit".

8. Voir la NZZ du 17 juin 1981. Cette motion discutée en juin 1981 au Conseil des Etats, fut renvoyée pour examen à sa Commission des Affaires étrangères. Les délibérations de cette dernière incitèrent Carlo Schmid à retirer sa motion lors de la session d'automne 1981 des Chambres fédérales.

9. II faut noter que les premiers en date des reproches adressés à la DDA dans cette vague de critiques sont venus - de façon assez surprenante - de l'administration elle-même ! En septembre 1980, Raymond Probst, le nouveau Secrétaire d'Etat du Département fédéral des affaires étrangères "reprend en main" (ce sont ses termes) le Département et, dans une interview accordée au Journal de Genève (12 septembre 1980) déclare : "Faute d'une coordination suffisante, on a vu se faire jour une sorte de doctrine à part, de politique propre au service de la coopération technique, allant jusqu'à contester la doctrine inspirant nos relations commerciales..." 
10. Le principal défenseur de cette thèse, Toni Hagen, fait une analyse manichéenne des prestations des secteurs public et privé dans le domaine de la coopération au développement - cf. NZZ du 4 mars 1981 - qui est surtout intéressante par l'écho qu'elle a rencontré auprès des critiques de la DDA.

11. Voir Tribune de Lausanne du 9 mai 1981. Ce chiffre élevé s'explique par la possibilité qu'ont les entreprises suisses de participer aux adjudications pour des projets financés par les banques de développement.

12. La campagne de presse contre la N.O. affirmant que la gestion des projets par la DDA était déficiente et que cette administration était hostile à l'économie privée est venue opportunément fournir des arguments aux revendications matérielles de certains secteurs de l'économie vis-à-vis de la coopération au développement.

13. Message concemant la continuation de continuation de la coopération technique et de l'aide financière en faveur des pays en développement du 9 juillet 1980 (FF 1980 II, p. 1357).

14. L'article 5 de la loi sur la coopération au développement et l'aide humanitaire internationales énonce ainsi les buts de la coopération au développement :

1. La coopération au développement soutient les efforts des pays en développement en vue d'améliorer les conditions de vie de leurs populations. Elle doit contribuer à mettre ces pays en mesure d'assurer leur développement par leurs propres forces. Elle tend, à long terme, vers un meilleur équilibre au sein de la communauté internationale.

2. Elle soutient en priorité les efforts des pays en développement, régions et groupes de populations les plus défavorisés. Elle encourage notamment :

a) Le développement rural.

b) L'amélioration alimentaire, en particulier les cultures vivrières destinées à la consommation locale.

c) La promotion de l'artisanat et de la petite industrie locale.

d) La création d'emplois. 
e) La recherche et le maintien d'un équilibre écologique et démographique. 